\title{
Cirugía despierta con mapeo cortico-subcortical en gliomas difusos adyacentes al lóbulo central. Reporte de dos casos y revisión de la literatura
}

\author{
Awake surgery with cortical-subcortical mapping in diffuse gliomas adjacent to central \\ lobe. Report of two cases and literature review
}

Santiago Núñez-Velasco, Javier Avendaño-Méndez-Padilla, Ricardo García-Iturbide, Claudio H. Pech-Cervantes, Cristian A. Molina-Martínez y Sonia Mejía-Pérez*

Departamento de Neurocirugía Oncológica, Instituto Nacional de Neurología y Neurocirugía Manuel Velasco Suárez, Ciudad de México, México

\begin{abstract}
Resumen
Introducción: Los gliomas difusos son neoplasias cerebrales con un patrón de crecimiento infiltrativo, frecuentemente adyacentes a áreas elocuentes. El mapeo cerebral con estimulación cortico-subcortical con el paciente despierto es una herramienta útil para lograr la mayor resección con el menor déficit posoperatorio. Presentación de casos: Varón de 24 años con crisis tónico-clónicas. La resonancia magnética (RM) mostró un glioma parietal izquierdo. Se realizó cirugía con el paciente despierto y mapeo cerebral por estimulación cortical y subcortical directa. Se obtuvo una resección casi total, ya que se encontraron áreas fucionales en el borde rostral del tumor. El paciente egresó sin déficit neurológico. Varón de 29 años que presenta crisis tónico-clónicas generalizadas, acompañadas de hemiparesia derecha. La RM reportó un glioma parietal izquierdo. Se realizó cirugía con el paciente despierto y mapeo cerebral por estimulación cortical y subcortical directa. Se logró una resección total y el paciente egresó sin déficit. Conclusiones: La cirugía con el paciente despierto con mapeo por estimulación directa y neuronavegación es la mejor opción en el tratamiento de los gliomas difusos, para lograr una resección máxima tumoral asegurando un mayor tiempo libre de enfermedad y la conservación de la función de áreas elocuentes.
\end{abstract}

PALABRAS CLAVE: Cirugía con paciente despierto. Estimulación cortical directa. Glioma Difuso. Mapeo cerebral. Neuronavegación.

\begin{abstract}
Introduction: Diffuse gliomas are brain neoplasms with an infiltrative growing pattern to cortical and subcortical structures, frequently adjacent to eloquent areas; direct cortical and subcortical stimulation in awake craniotomy is a useful tool to achieve a gross total resection with the least neurological deficit. Presentation of Cases: A 24 years old male presented with tonic-clonic seizures. The magnetic resonance imaging (MRI) showed a left parietal glioma. Awake craniotomy was performed using neuronavigation system and brain mapping with cortical and subcortical stimulation. Functional areas were found at the rostral margin of the tumor; however, the rest of the tumor was almost totally resected. Patient was discharged without neurological deficit. A 29 years old male presented in two occasions generalized tonic-clonic seizures, with right hemiparesis. The MRI showed a left parietal glioma. Awake craniotomy was performed using neuronavigation system and brain mapping with cortical and subcortical stimulation, achieving a gross total resection. Patient was discharged without neurological deficit. Conclusions: Awake craniotomy with brain mapping by cortical and subcortical stimulation and neuronavigation, are the best
\end{abstract}

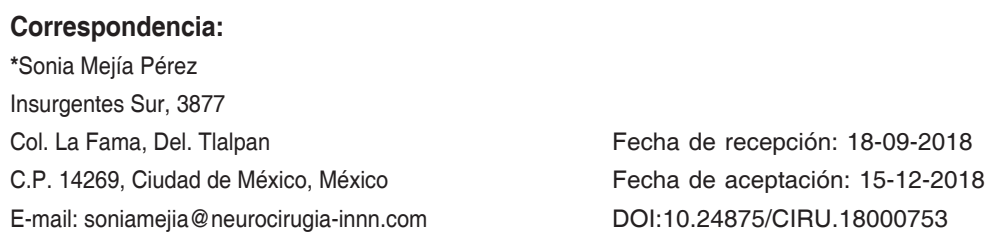


assets to treat diffuse gliomas and achieve a gross total resection, ensuring the major disease-free interval and preserving the function of eloquent areas.

KEY WORDS: Awake craniotomy. Brain mapping. Cortical stimulation. Diffuse gliomas. Neuronavigation.

\section{Introducción}

Los gliomas son neoplasias del sistema nervioso central que se nombran de acuerdo con la estirpe celular específica de la que se originan, y el grado que se les otorga corresponde a su agresividad biológica, siendo el grado IV el de mayor malignidad'. Son los tumores cerebrales intrínsecos más comunes, y su manejo terapéutico, tanto para los gliomas de bajo como de alto grado, se basa en realizar la máxima resección posible, debido a que la extensión de esta es uno de los factores más importantes que influyen en la supervivencia y en el periodo libre de enfermedad ${ }^{2,3}$. Sin embargo, la preservación de la función neurológica de los pacientes es de igual importancia, ya que no sólo influye en la supervivencia, sino también en la calidad de vida ${ }^{4,5}$.

Las lesiones en áreas elocuentes representan un reto particular debido a la variabilidad en la organización somatotópica cortical, la distorsión de la topografía cerebral resultante del efecto de masa y la reorganización funcional secundaria a la plasticidad ${ }^{6}$.

Entre las técnicas intraoperatorias que mayor utilidad tienen para preservar la funcionalidad se encuentra la cirugía con estimulación directa córtico-subcortical en el paciente despierto, así como la cirugía guiada con neuronavegación, las cuales pueden mejorar la resección en un 30-40\% sin comprometer la integridad funcional del paciente. El mapeo cerebral intraoperatorio de las funciones sensoriomotoras y de lenguaje es el método de referencia para la identificación y el cuidado de las áreas elocuentes, pudiendo alcanzar así la máxima resección segura ${ }^{6,7}$.

En el presente trabajo se hace el reporte de dos pacientes con diagnóstico de glioma difuso de bajo grado, ambos en íntima relación con el lóbulo central, que fueron sometidos a cirugía con el paciente despierto utilizando mapeo intraoperatorio mediante estimulación directa.

\section{Reporte de caso 1}

Varón de 24 años con único antecedente de consumo de alcohol, cannabis y cocaína, que acude a atención médica por presentar crisis tónico-clónicas generalizadas de difícil control con 5 meses de evolución, con déficit motor proporcionado 4/5 del hemicuerpo izquierdo con hipereflexia, sin más hallazgos en la exploración neurológica, con una puntuación en la escala de Karnosfky de 90. La resonancia magnética (RM) mostró una lesión difusa que involucra el lobulillo parietal superior izquierdo, infiltrando y desplazando el lóbulo central y ubicándose en íntima relación con este, con mínimo realce tras la administración de gadolinio, y edema vasogénico perilesional. La tractografía por imágenes de tensor de difusión (DTI) mostró desplazamiento rostral de la vía corticoespinal sin infiltración de la misma, así como compresión ventral de la porción retrolenticular de la capsula interna y su proyección. Se realizó medición de la masa tumoral por imagen, que reportó un volumen prequirúrgico de $53.03 \mathrm{~cm}^{3}$ (Fig. 1).

Se procedió a realizar cirugía con protocolo de paciente dormido, despierto, dormido, con apoyo de neuronavegación. Una vez hecha la craneotomía centrada en el sitio de la lesión y dejando un margen rostral de $2 \mathrm{~cm}$ para acceder al lóbulo central, se procedió a despertar al paciente y realizar mapeo por estimulación directa córtico-subcortical, iniciando con $2 \mathrm{~mA}$ y llegando hasta $8 \mathrm{~mA}$, encontrando zonas positivas específicamente de la mano y del brazo a $5 \mathrm{~mm}$ de la lesión. Se presentaron tres crisis motoras durante la estimulación directa que fueron yuguladas con solución salina helada (se congeló la solución y se administró tras su inmediato derretimiento), y pudo continuarse con el procedimiento. Una vez marcadas las zonas elocuentes se procedió a realizar la resección del tumor, que tenía características difusas sin márgenes identificables y de difícil aspiración. Al realizar mapeo subcortical en el borde rostral del tumor se encontró positividad en la pierna y la mano, por lo que se decidió parar la resección y dejar la porción elocuente de la lesión. Durante todo el proceso de extirpación se mantuvo comunicación constante con el paciente, pidiéndole que realizara tareas motoras (Fig. 2). El paciente egresó a recuperación, en donde no mostró ningún déficit agregado y fue egresado sin complicaciones a las 48 horas posquirúrgicas. A las 3 semanas de seguimiento se encuentra integrado a sus actividades habituales, con una puntuación de Karnofsky de 100 (Fig. 3) El patólogo reportó 


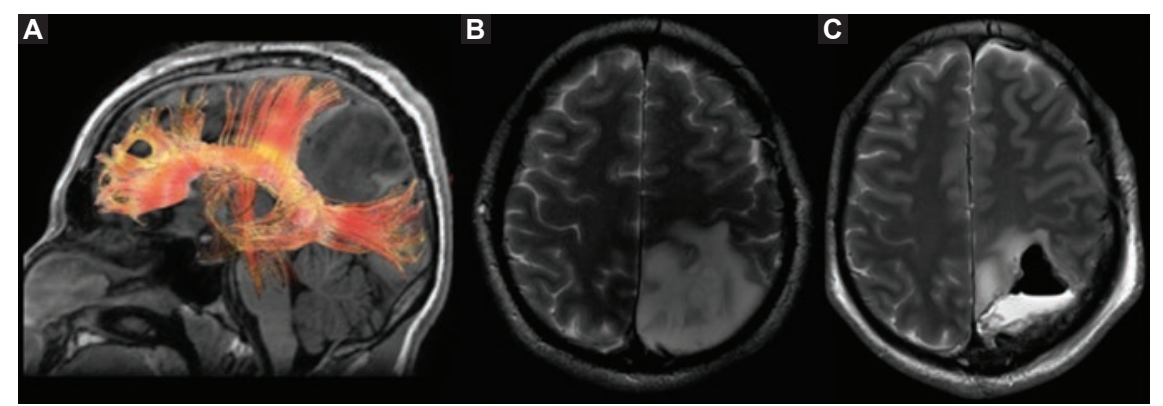

Figura 1. Caso 1. A: Tractografía por imágenes de tensor de difusión que muestra la proximidad del tumor con la vía córtico-espinal, desplazándola rostralmente y presionando de manera ventral las proyecciones retrolenticulares de la cápsula interna. B: Masa tumoral difusa ubicada en el lobulillo parietal superior en íntima relación con el lóbulo central en su porción rostral. C: Resonancia magnética posquirúrgica que muestra una adecuada resección con enfermedad residual (edema más lesión) justo en la zona que dio positivo en el mapeo subcortical.

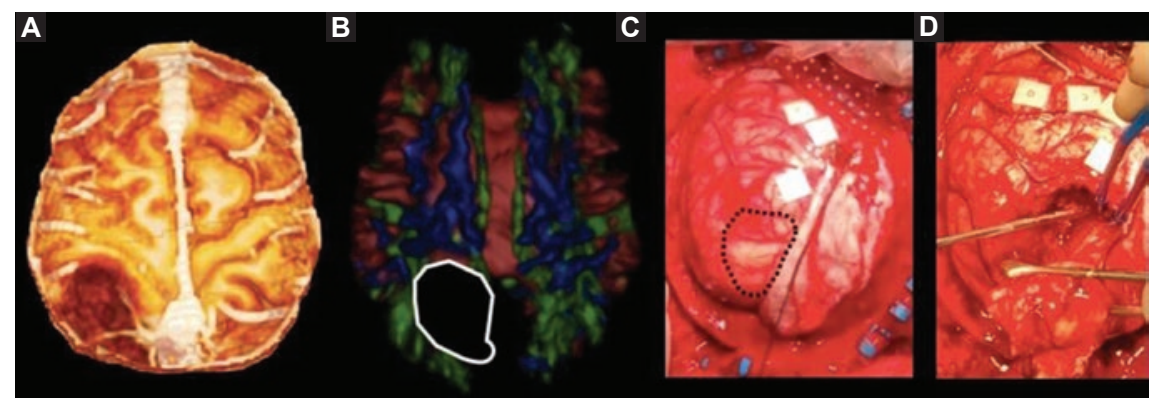

Figura 2. Caso 1. A: Reconstrucción volumétrica de resonancia magnética que muestra una tumoración difusa del lobulillo parietal superior, con compresión rostral del lóbulo central. B: Tractografía que muestra la cercanía de la lesión con la vía córtico-espinal (azul). C: Imagen transquirúrgica que muestra en línea punteada la zona de la lesión con las zonas positivas en el mapeo cortical por estimulación directa córtico-subcortical señalizadas. D: Durante la resección, nótese que se respetan las áreas corticales mapeadas y marcadas con letras.

astrocitoma difuso sin determinación molecular (NOS) de grado II según la Organización Mundial de la Salud (OMS).

\section{Reporte de caso 2}

Varón de 29 años, con único antecedente de tabaquismo suspendido un año antes del diagnóstico. Acude a nuestra institución por presentar dos crisis tónico-clónicas generalizadas de 5 meses de evolución, así como parestesias ocasionales del hemicuerpo derecho. A la exploración no presenta ningún déficit neurológico, con una puntuación en la escala de Karnosfky de 90. La RM (Fig. 4) mostró una lesión difusa del lobulillo parietal superior izquierdo en íntima relación con el lóbulo central, comprimiéndolo y desplazándolo rostralmente, con edema vasogénico perilesional, sin realce tras el medio de contraste. La DTI mostró desplazamiento rostral de la vía corticoespinal adyacente a la lesión, sin infiltración de la misma y desplazamiento lateral del fascículo longitudinal superior II. Se realizó medición de la masa tumoral por imagen, que reportó un volumen prequirúrgico de $42.32 \mathrm{~cm}^{3}$ (Fig. 4).

Se realizó cirugía con protocolo de paciente dormido, despierto, dormido, con apoyo de neuronavegación.
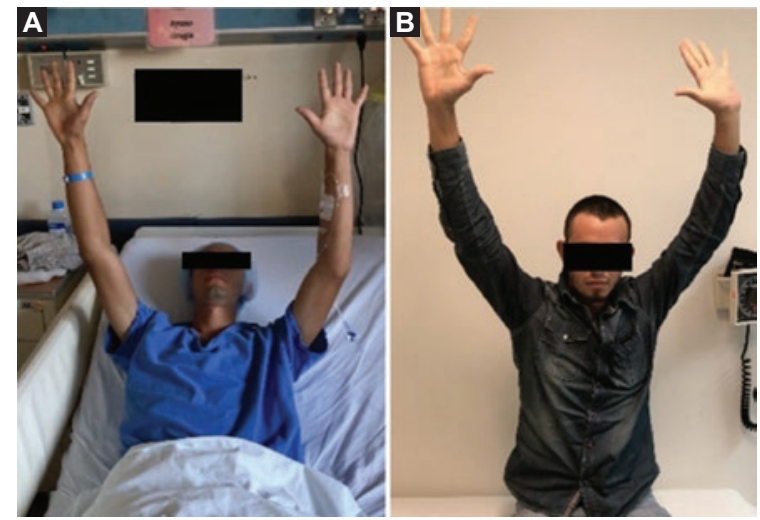

Figura 3. A: Exploración motora de las extremidades superiores antes de entrar al procedimiento quirúrgico. B: Exploración motora de las extremidades superiores 3 semanas después de la cirugía.

Una vez realizada la craneotomía centrada en la lesión se despertó al paciente para iniciar la estimulación directa córtico-subcortical con 2 a $8 \mathrm{~mA}$, localizando las áreas motoras de la mano y el brazo a $1 \mathrm{~mm}$ del margen rostral del tumor. Se presentó una crisis motora durante el mapeo, que fue yugulada con solución helada y pudo continuarse con el procedimiento. Una vez mapeadas las áreas elocuentes se procedió a la resección, pidiendo en todo momento que el paciente realizara tareas motoras. La estimulación subcortical fue negativa, por 

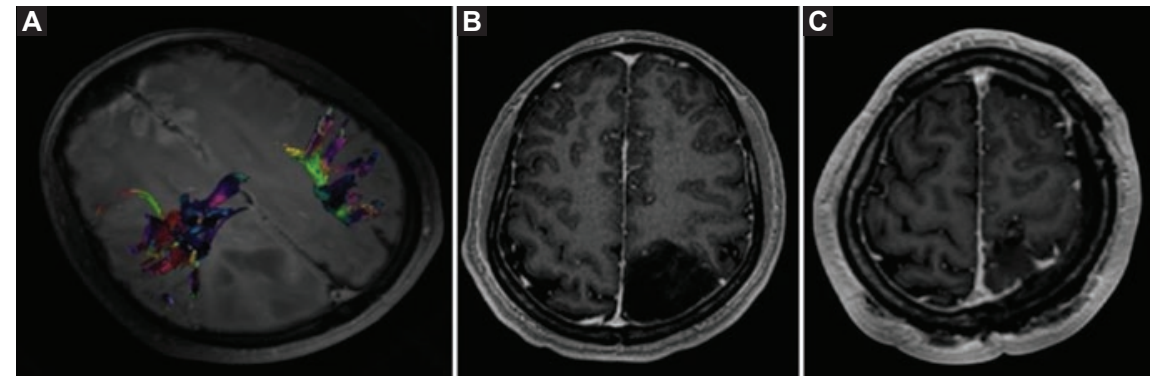

Figura 4. Caso 2. A: Se observa en la tractografía por imágenes de tensor de difusión la relación del tumor difuso con la vía piramidal, la cual se encuentra desplazada en sentido rostral (azul), y también puede verse el fascículo longitudinal superior II, desplazado en sentido lateral, ambos muy cerca del tumor. B: Resonancia magnética prequirúrgica que muestra una lesión tumoral difusa del lobulillo parietal superior que infiltra el lóbulo central izquierdo. C: Resonancia magnética posquirúrgica que muestra el área de resección.
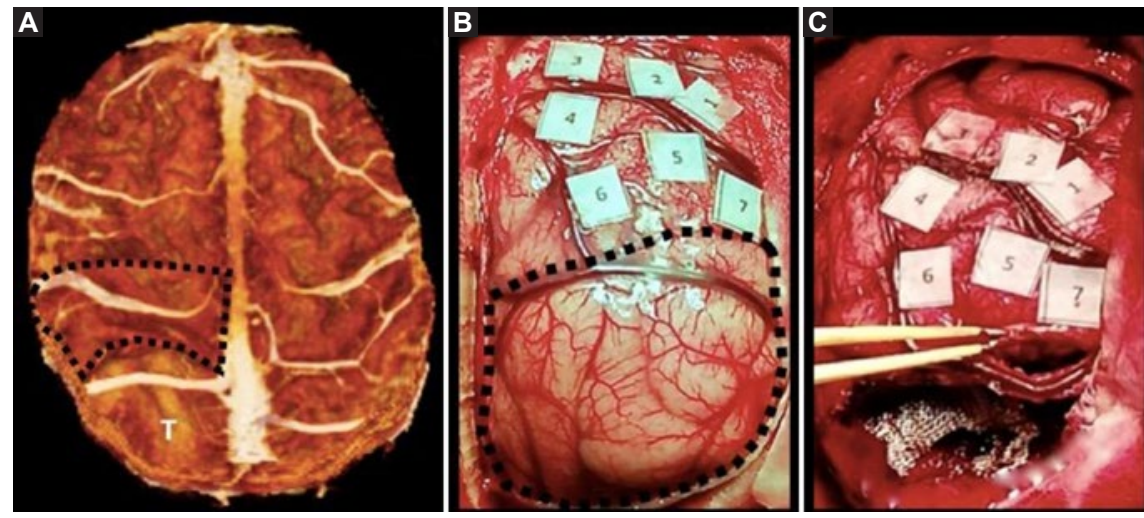

Figura 5. Caso 2. A: Reconstrucción volumétrica de resonancia magnética en la que se observa el lóbulo central (línea punteada); nótese la íntima relación con el tumor (T). Compromiso del giro parietal superior y desplazamiento del lobulillo central. B: Mapeo por estimulación directa córtico-subcortical transoperatoria con delimitación de áreas elocuentes previa a la resección (en línea punteada, la lesión tumoral). C: Se observa el drenaje venoso superficial íntegro, así como las áreas córtico-subcorticales mapeadas después de la resección.

lo que fue posible acceder a las zonas más rostrales del tumor (Fig. 5). El paciente egreso a recuperación sin déficit motor agregado, pero con parestesias en el brazo izquierdo. Fue egresado sin complicaciones a las 48 horas posquirúrgicas. A las 3 semanas de seguimiento, el paciente no presenta ningún déficit, las parestesias han cedido y su puntuación de Karnosfky es de 100 (Fig. 6). El reporte histopatológico fue de oligoastrocitoma difuso NOS de grado II de la OMS.

En ambos casos se consideró someter a cirugía con paciente despierto debido a que eran jóvenes con vidas activas, con una puntuación en la escala de Karnosfky de 90 y por RM presentaban un tumor intrínseco supratentorial adyacente a regiones sensoriales y motoras del lóbulo central. La estimulación cortical y subcortical directa fue bipolar, con $5 \mathrm{~mm}$ de distancia entre las puntas, frecuencia de pulso de $60 \mathrm{~Hz}, 1 \mathrm{~ms}$ de duración del pulso, amplitud bifásica de 2-8 mA y 4 segundos de contacto. Los sitios positivos y negativos se verificaron en tres ocasiones. Los porcentajes de resección tumoral reportados por RM fueron del $80.1 \%$ en el caso 1 y del $98 \%$ en el

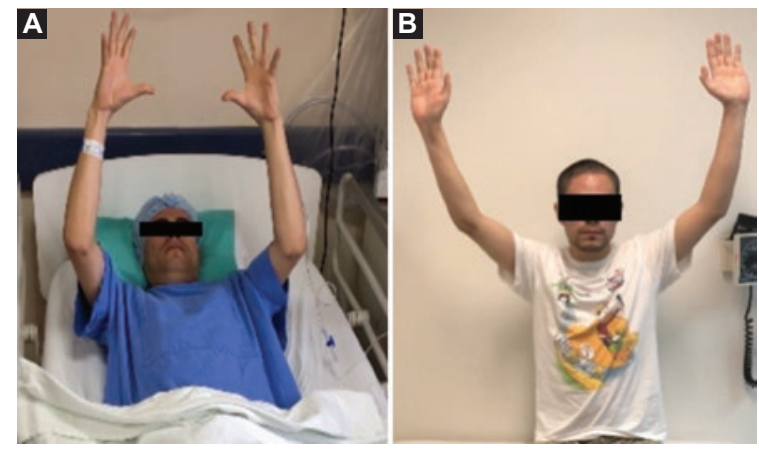

Figura 6. A: Exploración motora de las extremidades superiores antes de entrar al procedimiento quirúrgico. B: Exploración motora de las extremidades superiores 3 semanas después de la cirugía.

caso 2; en ambos se confirmó haber realizado una resección máxima segura.

Los dos pacientes firmaron su consentimiento para aparercer en este reporte.

\section{Discusión}

La OMS, en su última clasificación de tumores del sistema nervioso central del año 2016, agrupa los 
tumores cerebrales de acuerdo con sus características histológicas, moleculares y de comportamiento, por lo que en el grupo de gliomas difusos se encuentran el astrocitoma difuso de grado II, el astrocitoma anaplásico de grado III, el oligoastrocitoma de grado II, el oligoastrocitoma anaplásico de grado III y el glioblastoma de grado IV. Los astrocitomas anaplási$\cos$ y difusos representan el $25 \%$ de los gliomas difusos ${ }^{8,9}$. En cuanto al periodo de sobrevida postratamiento, se reportan 78.1 meses para los gliomas de bajo grado, 37.6 meses para los gliomas anaplásicos y 14 meses para los glioblastomas ${ }^{9,10}$.

El patrón de crecimiento temprano del glioma consta de tres fases distintas: la fase I se caracteriza por células gliomatosas confinadas al sitio inicial de implantación; en la fase II la morfología cambia al desplegarse cadenas de elongaciones celulares fuera del sitio de implantación, asumiendo una estructura en forma de rama; y en la fase III el centro se vuelve denso a la vez que aumenta la masa tumoral y mantiene un patrón invasor ${ }^{11}$. La transición de un tumor de tipo benigno a uno de tipo maligno se caracteriza por una secuencia de fenotipos, siendo la tasa de proliferación celular y la velocidad de migración celular los factores clave. Siguiendo el principio go or grow, mientras las células altamente invasivas tienen una tasa de proliferación baja, las altamente proliferativas son menos invasoras ${ }^{12,13}$.

Con estas cualidades tumorales y con la persistencia de células invasoras en los márgenes tumorales se dificulta diferenciar, tanto en los estudios de imagen como en la resección, entre tejido sano y neoplásico, además de representar un reto clínico debido al diagnóstico tardío y a las escasas opciones terapéuticas disponibles al momento de realizarlo ${ }^{14,15}$. Por ese motivo, el manejo terapéutico debe ser multidisciplinario y requiere neuroimagen, cirugía, neuropatología, radioterapia y quimioterapia ${ }^{9}$.

Las localizaciones más frecuentes de invasión tumoral son el lóbulo frontal (30\%), el área motora suplementaria $(27 \%)$ y la ínsula $(25 \%)^{16}$. De forma tradicional se ha aceptado que los giros precentral y poscentral son áreas con funciones diferentes, pero se ha demostrado que estas se superponen y que ambos giros no están simplemente divididos por el surco central. En otras palabras, esto implica que la invasión de gliomas en estas áreas puede causar síntomas tanto sensitivos como motores, mixtos o arrestos del movimiento. Por ello, el Dr. Gazi Yaşargil acuñó el término «lóbulo central» para agrupar al giro frontal ascendente y al parietal ascendente como una unidad funcional que hace que sea aún más importante su mapeo durante la cirugía ${ }^{17}$.

El estudio de elección para el diagnóstico de estos tumores es la RM. Una de las secuencias con mayor utilidad es la DTI, que se ha considerado la mejor técnica no invasiva preoperatoria para identificar el trayecto de las fibras blancas adyacentes a la lesión. En los casos reportados en este artículo, así como en la publicación de Castellano, et al. ${ }^{18}$, el uso prequirúrgico de tractografía contribuyó de forma positiva en la planeación de una mejor estrategia quirúrgica para la resección, aumentando el área de resección al visualizar los trayectos de las fibras desplazadas sin infiltración $n^{7,18,19}$.

Aunado a esto, se utilizan herramientas como la neuronavegación, que basada en estudios de gabinete previos a la cirugía genera impresiones individualizadas de la relación entre las áreas funcionales y la lesión tumoral, facilitando la resección al permitir visualizar los límites tumorales en el momento quirúrgico ${ }^{4,19,20}$.

La cirugía en pacientes con gliomas difusos tiene como objetivos el diagnóstico, maximizar la resección, minimizar los riesgos de transformación maligna y aumentar el tiempo medio de supervivencia manteniendo la calidad de vida de los pacientes ${ }^{9,20}$. Esto se logra con la estimulación directa cortical o subcortical, que se considera el método de referencia para mapear los límites funcionales de tumores cerebrales. Esta se lleva a cabo con un electrodo bipolar; la estimulación producirá un movimiento clónico con contracción tónica del área estimulada, permitiendo diferenciar entre las áreas elocuentes y las que no lo son. Debido a la probabilidad de generar crisis convulsivas inducidas por la estimulación, es necesario tener disponible solución cristaloide para disminuir la reacción cerebral por el estímulo generado ${ }^{6}$. Con el uso de mapeo por estimulación directa cortical y subcortical se ha demostrado una reducción de la morbilidad neurológica posquirúrgica a menos del 1.5\%,4,21,22. En un metaanálisis realizado por De Witt Hamer, et al. ${ }^{23}$ en 2012 se concluyó que el mapeo intraoperatotrio se asocia con una reducción dos veces mayor de presentar déficits neurológicos tardíos en comparación con no realizar dicho mapeo, además de lograr una mayor extensión de la resección tumoral de las lesiones que involucran áreas elocuentes. Otras herramientas, como la RM intraoperatoria, han demostrado ser útiles para lograr resecciones más amplias, pero no garantizan que se respeten las áreas elocuentes durante la cirugía y el tiempo de realización de la RM puede ser de hasta 30 minutos, por lo que es dificil mantener al paciente 
despierto y tranquilo durante el mapeo. Barbagallo, et al. ${ }^{24}$ reportan una serie de casos utilizando la tomografía intraoperatoria para verificar la máxima resección posible y ajustar la navegación, ya que después de iniciar la resección por la pérdida de volumen se genera el fenómeno llamado brain shift, que hace que el registro del navegador sea menos preciso. En este estudio se utilizó el mapeo con paciente despierto para verificar las áreas funcionales. Selbekk, et al. ${ }^{25}$ reportan el ultrasonido transquirúrgico como otra herramienta para verificar el volumen de resección y ajustar el brain shift del navegador, pero con el ultrasonido transquirúrgico tampoco es posible asegurar que se respetan las áreas funcionales.

En nuestro estudio se utiliza DTI para determinar si las fibras subcorticales se encuentran infiltradas 0 desplazadas, y en su caso en qué dirección, y la neuronavegación para la planeación inicial del abordaje y la ubicación del tumor, pero una vez iniciada esta, es con el mapeo directo córtico-subcortical como aseguramos que no se lastimarán áreas elocuentes (como se muestra en el primer caso, en el que se interrumpió la resección por encontrar zonas postivas en el mapeo a pesar de que el DTI mostraba el fascículo corticoespinal libre de infiltración), ya que según los estudios previamente descritos es la herramienta más precisa para lograr la máxima resección funcional posible, recomendando ampliamente (si se tienen disponibles) la combinación del ultrasonido transquirúrgico y la tomografía intraoperatoria con el mapeo directo para lograr mejores resultados.

\section{Conclusiones}

Los gliomas difusos tienen un patrón de invasión significativo a áreas elocuentes. En estos casos, el lóbulo central constituye una unidad funcional con áreas tanto motoras como sensitivas mezcladas, y no francamente delimitadas como en las descripciones anatómicas clásicas. Por este motivo, la individualización en el abordaje con ayuda de los estudios de gabinete, como la RM y la tractografía por DTI, permite tanto un mayor entendimiento de las alteraciones provocadas en la neuroanatomía de cada paciente como una mejor planeación del procedimiento quirúrgico con el propósito de lograr una cirugía más segura. Las técnicas usadas durante el procedimiento quirúrgico, como el mapeo cerebral con estimulación directa cortical y subcortical, y la neuronavegación, son fundamentales para lograr una mayor resección tumoral y disminuir las complicaciones neurológicas posquirúrgicas.

\section{Financiamiento}

La elaboración de este trabajo original no requirió financiamiento.

\section{Conflicto de intereses}

No existen conflictos de intereses.

\section{Responsabilidades éticas}

Protección de personas y animales. Los autores declaran que para esta investigación no se han realizado experimentos en seres humanos ni en animales.

Confidencialidad de los datos. Los autores declaran que han seguido los protocolos de su centro de trabajo sobre la publicación de datos de pacientes.

Derecho a la privacidad y consentimiento informado. Los autores han obtenido el consentimiento informado de los pacientes o sujetos referidos en el artículo. Este documento obra en poder del autor de correspondencia.

\section{Bibliografía}

1. Louis DN, Perry A, Reifenberger G, von Deimling A, Figarella-Branger D, Cavenee WK, et al. The 2016 World Health Organization classification of tumors of the central nervous system: a summary. Acta Neuropathol. 2016;131:803-20.

2. Ius T, Isola M, Budai R, Pauletto G, Tomasino B, Fadiga L, et al. Low-grade glioma surgery in eloquent areas: volumetric analysis of extent of resection and its impact on overall survival. A single-institution experience in 190 patients. J Neurosurg. 2012;117:1039-52.

3. Hervey-Jumper S, Li J, Lau D, Molinaro A, Perry D, Meng L, et al. Awake craniotomy to maximize glioma resection: methods and technical nuances over a 27-year period. J Neurosurg. 2015;123:325-39.

4. Hervey-Jumper S, Berger M. Maximizing safe resection of low and high grade glioma. J Neurooncol. 2016;130:269-82.

5. Sanai N, Berger MS. Glioma extent of resection and its impact on patient outcome. Neurosurgery. 2008;62:753-66.

6. Sanai N, Mirzadeh Z, Berger M. Functional outcome after language mapping for glioma resection. N Engl J Med. 2008;358:18-27.

7. Krivosheya D, Prabhu S, Weinber J, Sawaya R. Technical principles in glioma surgery and preoperative considerations. J Neurooncol. 2016;130:243-52.

8. Louis DN, Ohgaki H, Wiestler OD, Cavenee WK, editores. WHO classification of tumors of the central nervous system. Lyon: IARC Press; 2007.

9. Jiang T, Mao Y, Ma W, Mao Q, You Y, Yang X, et al. CGCG clinical practice guidelines for the management of adult diffuse gliomas. Cancer Lett. 2016;375:263-73.

10. Duffau H. Diffuse low-grade gliomas and neuroplasticity. Diag Interv Imaging. 2014;95:945-55.

11. Baker G, Nand V, Motsch S, Koschmann C, Calinescu A, Mineharu Y, et al. Mechanisms of glioma formation: iterative perivascular glioma growth and invasion leads to tumor progression, VEGF-independent vascularization, and resistance to antiangiogenic therapy. Neoplasia. 2014;16:543-61.

12. Hatzikirou H, Basanta D, Simon M, Schaller K, Deutsch A. 'Go or grow': the key to the emergence of invasion in tumor progression? Math Med Biol. 2012;29:49-65.

13. Xie $Q$, Mittal S, Berens $M$. Targeting adaptive glioblastoma: an overview of proliferation and invasion. Neuro-Oncol. 2014;16:1575-84.

14. Esmaeili M, Stensjøen, Berntsen E, Solheim O, Reinertsen I. The direction of tumor growth in glioblastoma patients. Sci Rep. 2018;8:1199.

15. Prastawa M, Bullit E, Gerig G. Simulation of brain tumor in MR images for evaluation of segmentation efficacy. Med Image Anal. 2009;13:297-311.

16. Darlix A, Gozé C, Rigau V, Bauchet L, Taillandier L, Duffau H. The etiopathogenesis of diffuse low-grade gliomas. Crit Rev Oncol Hematol. 2017; 109:51-62. 
17. Nii Y, Uematsu S, Lesser RP, Gordon B. Does the central sulcus divide motor and sensory functions? Cortical mapping of human hand areas as revealed by electrical stimulation through subdural grid electrodes. Neurology. 1996;46:360-7

18. Castellano A, Bello L, Michelozzi C, Galluci M, Fava E, ladanza A, et al Role of diffusion tensor magnetic resonance tractography in predicting the extent of resection in glioma surgery. J Neurooncol. 2012; 14:192-202.

19. Motomura K, Chalise L, Onka F, Aoki K, Tanahashi K, Hirano M, et al. Supratotal resection of diffuse frontal lower grade gliomas with awake brain mapping, preserving motor, language, and neurocognitive functions. World Neurosurg. 2018;119:30-9.

20. Sang S, Wanggou S, Wang Z, Lin X, Jiang N, Ye N. Clinical long-term follow-up evaluation of functional neuronavigation in adult cerebral gliomas. World Neurosurgery. 2018;119:e262-71.

21. Han SJ, Morshed RA, Troncon I, Jordan KM, Henry RG, Hervey-Jumper SL, et al. Subcortical stimulation mapping of descending motor pathways for perirolandic gliomas: assessment of morbidity and functional outcome in 702 cases. J Neurosurg. 2018 Aug 1:1-8. doi: 10.3171/2018.3.JNS172494. [Epub ahead of print]

22. Pallud J, Rigaux-Viode O, Corns R, Muto J, Lopez Lopez C, Mellerio C, et al. Direct electrical bipolar electrostimulation for functional cortical and subcortical cerebral mapping in awake craniotomy. Practical considerations. Neurochirurgie. 2017;63:164-74.

23. De Witt Hamer PC, Robles SG, Zwinderman AH, Duffau H, Berger MS. Impact of intraoperative stimulation brain mapping on glioma surgery outcome: a meta-analysis. J Clin Oncol. 2012;30:2559-65.

24. Barbagallo GMV, Morrone A, Certo F. Intraoperative computed tomography and awake craniotomy: a useful and safe combination in brain surgery. World Neurosurg. 2018;119:e159-66.

25. Selbekk T, Jakola AS, Solheim O, Johansen TF, Lindseth F, Reinertsen I, et al. Ultrasound imaging in neurosurgery: approaches to minimize surgically induced image artefacts for improved resection control. Acta Neurochirur (Wien). 2013;155:973-80. 